\title{
Leveling the Playing Field: An Interprofessional Rural Preceptorship Pilot for Students of Nursing and Medicine
}

\author{
Deirdre Jackman ${ }^{1}$, Olive Yonge ${ }^{1,}$, Florence Myrick ${ }^{1}$, Fred Janke ${ }^{2} \&$ Jill Konkin $^{3}$ \\ ${ }^{1}$ Faculty of Nursing, University of Alberta, Canada \\ ${ }^{2}$ Department of Family Medicine; Director of Rural and Regional Health, Faculty of Medicine and \\ Dentistry, University of Alberta, Canada \\ ${ }^{3}$ Division of Community Engagement, Faculty of Medicine and Dentistry, University of Alberta, \\ Canada \\ *Corresponding author: Faculty of Nursing, University of Alberta, Canada. E-mail: \\ djackman@ualberta.ca \\ Received: January 9, 2018 Accepted: January 22, 2018 Published: January 24, 2018 \\ doi:10.5296/jsr.v9i1.12456 URL: https://doi.org/10.5296/jsr.v9i1.12456
}

\begin{abstract}
Despite the ubiquity of interprofessional education (IPE) in the health sciences, cultural barriers to collaboration and socialization across disciplines are pervasive and persistent. The Interprofessional Rural Preceptorship Pilot (IPRPP) was implemented over 18 months between 2014 and 2015, to deliver mutual, practice-based, collaborative experiences to students of nursing and medicine, and to capitalize on the multidisciplinary team ethos of rural settings in socializing these students into an interprofessional culture. Students; precepting nurses and physicians; unit managers; and faculty supplied qualitative research data through semistructured interviews and focus groups, to help determine the effectiveness of the IPRPP in achieving its ends. Thematic analysis of transcripts, field notes and memos revealed that the pilot: 1) induced all participants to reflect on cultural barriers to IPE, particularly in light of the more experienced, male physicians' apparent reluctance to buy into the pilot or the research study; 2) sensitized medical students to the scope of practice, roles and knowledge base of registered nurses and nursing students, whom they came to value as collaborators and teachers; and 3) inspired nursing students to take the lead in arranging interprofessional experiences, and to assert their viewpoints as equals within the multidisciplinary team. It was found that liminal (transitional) spaces and the frontline relaxation of traditional hierarchies - both widely acknowledged features of rural health care settings - contributed to these outcomes. The findings show that clinical settings have a significant impact on the interprofessional socialization of nursing and medical students, with implications for the design of future IPE initiatives.
\end{abstract}

Keywords: Interprofessional education, Multidisciplinary teams, Preceptorship, Rural nursing, Rural medicine, Thematic analysis 


\section{Introduction}

Interprofessional education (IPE) has been a fixture of health sciences curricula for over a generation, signifying widespread recognition that patients' needs and health care team ethos require a shift away from the traditional segregation of disciplines (McNair, 2005; WHO, 2010). The goal of IPE is to foster teamwork between caregivers from distinct professions, through mutual, interactive learning (Reeves et al., 2013). It is equally apparent, however, that cultural barriers to collaboration between health care disciplines are deeply ingrained, despite the ubiquity of IPE. Profession-centric silos across health care disciplines continue to compromise quality of care and patient safety (Margalit et al., 2009; Price, Doucet, \& Hall, 2014; Weller, Boyd, \& Cumin, 2014; WHO, 2010).

Especially problematic is the hierarchical disconnect between nursing and medicine, wherein nurses' opinions and skillsets are marginalized by physicians, either overtly or through the actualization of a tacit, hierarchical discourse by both parties (McNair, 2005). Classroomand theory-based IPE interventions can do little to inoculate students in these disciplines against these pervasive and persistent cultural barriers in health care workplaces (Paradis \& Whitehead, 2015). The researchers set out to determine if collaborative, practice-based experiences, wherein students of nursing and medicine could undergo precepted, clinical rounds together, would have an appreciable effect on these students' attitudes, knowledge and behaviours pertaining to interprofessional (IP) teamwork.

Over an eighteen-month period between 2014 and 2015, the researchers implemented an Interprofessional Rural Preceptorship Pilot (IPRPP) involving students of nursing and medicine at a postsecondary institution in Western Canada. The pilot enabled these students to undertake clinical rotations side-by-side, and to interact with each other's preceptors. Chief features of the IPRPP were 1) the partnering of senior nursing students, about to graduate, with $3^{\text {rd }}$ Year medical students; and 2) rural acute and community care settings, which have been shown to immerse students in a collaborative culture of relaxed professional boundaries (Hays, 2008). Through thematic analysis of semi-structured interviews and focus group data, the researchers sought to address the research question: "How can a rural, IP preceptorship influence the attitudes, knowledge and behaviours of nursing and medical students, regarding IP communication and collaboration?" This article describes outcomes of the pilot as they pertain to this research question, and their implications for IPE.

\section{Background}

\subsection{Cultural Barriers to IPE}

Culturally entrenched power relationships among different health care professions influence practitioners' receptiveness to IPE initiatives (Baker et al., 2011; Becker, Hanyok \& Walton-Moss, 2014; Pecukonis, Doyle, \& Bliss, 2008; Whitehead, 2007). Older clinical instructors/preceptors and faculty, having themselves undergone a traditional, uniprofessional programme of study, are more apt to impart traditional, hierarchical values - specifically the "turf and baggage" of nursing and medicine-to their students (Becker, Hanyok \& 
Walton-Moss, 2014, p. 240). That these two professions remain stubbornly gendered (Seenandan-Sookdeo, 2012), particularly in the underrepresentation of women in senior medical positions (Bell, Michalec, \& Arenson, 2014), is an additional barrier to collaboration and IPE. Inroads in IPE notwithstanding, nurses and other members of the IP team still act out a normative discourse in which nurses have a lower status than other team members (Hart, 2015; Price, Doucet, \& Hall, 2014; Price, Hall, Angus, \& Peter, 2013; Seenandan-Sookdeo, 2012), with negative implications for the socialization of students on practicum.

Kuper and Whitehead (2012) question the effectiveness of IPE in promoting collaboration, insofar as physician-educators have a vested interest in maintaining the status quo. Interprofessionalism is a perceived threat to physicians' authority over other team members, thus physicians remain "largely unengaged in IPE activities" or simply employ the language of IPE in lieu of any actual reform to practice (p. 348). It is unclear whether Kuper and Whitehead (2012) take into account alternative models of IPE, such as practice-based initiatives. These authors do pertinently remark, "unfortunately, most of the rest of the IPE literature pays very little overt attention to power" (p. 348). Paradis and Whitehead (2015), in a comprehensive review of IPE literature, are more direct in criticizing a systemic blind spot to the hierarchical, disciplinary disconnects IPE purports to address:

Health professions educators and leaders are shirking responsibility if they give up on currently practising generations of health care providers, cobble together an IPE curriculum and expect that a sprinkling of fresh faces with more egalitarian ideas will change the system for the better. The fact that power and conflict are absent from the vast majority of articles written about IPE suggests that educators and researchers are hesitant to engage with the difficult yet undeniable truth that power structures shape health systems and health professional interactions (p. 405).

Here again, the authors appear to regard IPE as exclusively classroom-based, and do not speculate how setting contributes to the deficits they point out, nor does their review consider alternative models of IPE.

Students may adopt the stereotypical stances of their chosen disciplines towards IP collaboration, even before they undertake their programmes of study. Horsburgh et al. (2006) find that pre-medical students are much less likely to embrace a collaborative, team-based approach to clinical work than their pre-nursing counterparts. Moreover, siloed, hierarchical discourses are no less prevalent in educational settings than they are within professional health care teams. Students immersed in the logical-positivist, evidence-based culture of medical school are inherently sceptical about the cultural, qualitative aspects of IPE (Page \& Meerabeau, 2004), and may be less predisposed to take part in IPE research than students in other health care disciplines (Olson, 2015).

\subsection{Socialization and IPE Environment}

Price, Hall, Angus, and Peter (2013) hold out hope for the potential of "future recruitment initiatives, socialization strategies, and orientation programs"-albeit unspecified-“"to enhance IP collaboration within the health professions" (p. 513). The authors do pointedly 
distinguish such initiatives from traditional, classroom-based IPE, which they nonetheless also regard as essential. Weller, Boyd and Cumin (2014) propose a number of measures designed to enhance IP communication and reduce tribalism, including side-by-side training of health care team members.

Khalili et al. (2013) submit that IPE must be complemented by an interprofessional socialization (IPS) process whereby students in the health sciences mutually confront their insular, tribal tendencies; learn collaboratively; and build IP identities alongside their uniprofessional ones, all within a safe space for critical reflection and dialogue. While learning environment is clearly central to IPS, these authors leave open the question as to whether clinical practice settings can be ideal sites for the construction of IP identity. Physical setting is a key factor in the relaxation of power relationships between physicians and other health care professionals, as Bleakley (2013) points out. "Liminal" or transitional spaces within the health care setting, such as corridors, allow for communication between these disparate disciplines, unburdened by formal protocols (p. 28). This sitedness of hierarchical, IP discourse has obvious implications for IPS.

Another democratizing effect of clinical practice settings can be the "pragmatic hierarchies" that grow out of informal mentoring relationships between distinct disciplines (Burford et al., 2013, p. 397). It is widely known yet seldom acknowledged, for instance, that experienced nurses play an important role in educating and socializing novice physicians, in spite of the traditional, disciplinary hierarchy (Burford et al., 2013). Such relationships are borne of frontline necessity, rather than any formal program of IPS. However, such frontline, democratizing conditions present a clear opportunity for the construction of IP identities amongst students of nursing and medicine. Page and Meerabeau (2004) observe "the most favourable situation for (undergraduate) multiprofessional education occurs when the students have about the same level of educational background" (p. 122), implying that a level playing field, in terms of experience, helps to socialize students into an IP culture.

From the literature it is apparent that health sciences faculties and clinical practice settings are rife with cultural barriers to IPE, but the latter present opportunities for IPS when certain conditions - liminality of space, frontline relaxation of traditional hierarchies - are present. Rural practice settings have been found to meet these conditions (Hays, 2008), making it a worthwhile endeavour to explore the potential of these sites for practice-based IPE involving students of nursing and medicine, and the power of these conditions to support IP identity construction.

\section{Method}

\subsection{Study Aims}

The Interprofessional Rural Preceptorship Pilot (IPRPP) was implemented by members of the research team over 18 months, between 2014 and 2015. The pilot was designed to deliver IP practice experiences to nursing and medical students undertaking clinical preceptorships, while capitalizing on the informal, collaborative ethos of rural health care sites, thereby 
socializing these students into an IP practice culture. In addition to side-by-side clinical rounds, the IPRPP brought the students together for educational modules, both onsite and online, comprising the themes of Teaching and Learning; The Rural Context; Communication and Collaboration; and Roles and Responsibilities. Each module lasted approximately one hour and comprised case studies for discussion, video presentations, readings and quizzes.

While conducting the IPRPP, the researchers sought to address a central, underlying research question: "How can a rural, IP preceptorship influence the attitudes, knowledge and behaviours of nursing and medical students, regarding IP communication and collaboration?" To this end, the researchers engaged participating students, preceptors and faculty in semistructured, one-on-one interviews and focus groups. Findings emerged from thematic analysis of the research data, which comprised transcripts, memos and field notes.

\subsection{Participants}

Purposive and snowball sampling were employed in this study. Nursing and medical students at a western Canadian postsecondary institution were recruited through a faculty coordinator, unaffiliated with the research team. Five senior undergraduate nursing students, all female, aged 21-25; and five medical students, three male and two female, aged 24-32, agreed to take part in the study. The nursing students were undergoing their final preceptorship prior to graduation, while the medical students were undergoing a 3rd-year clinical rotation. None of the students had any prior clinical experience in a rural setting. Every student volunteered to participate in the IPRPP and the associated, qualitative research study.

Upon the students' placement at three rural health care sites (described below), the researchers went on to recruit six registered nurses (RNs), all female, aged 45-60, each having greater than 15 years' experience in rural practice; and six physicians (MDs), two female and four male, aged 29-60, each having between one and 27 years' rural practice experience. Each of these practitioners was assigned to precept one or more of the students already recruited. With the exception of two MDs, all preceptors had previous, clinical teaching experience. Additionally, three rural site managers and the nursing faculty coordinator provided interview data for the study. Three male MDs, aged 45-60, did not respond to interview or focus group invitations after the project orientation.

\subsection{Research Settings}

Student placements were spread across three sites: a 76-bed acute care setting in a semirural community (population 17,200), $95 \mathrm{~km}$ distant from the nearest major urban centre; a 10-bed acute care setting in a rural community (population 4400), $365 \mathrm{~km}$ distant from the nearest major urban centre; and a 17-bed acute care setting in a rural community (population 1,040), $136 \mathrm{~km}$ distant from the nearest major urban centre. Nursing preceptorships were 10 weeks in duration, while the medical rotations lasted eight weeks. The students also undertook rounds in the community primary care networks, long-term care facilities, and medical clinics where the precepting MDs practiced. 


\subsection{Ethical Considerations}

Before commencing the study, the researchers received approval from their employer's human research ethics board, the regional health authorities overseeing the sites where data collection was carried out, and the local managers of these sites. Following onsite orientation, all participants received a project information sheet and gave signed, informed consent, having been apprised of the study design and objectives, and their rights to remain anonymous and/or to withdraw at any point during the study. Pseudonyms have been employed to protect participants' confidentiality.

\subsection{Data Collection and Analysis}

The pilot took place in two phases, comprising the Winter-Spring terms in 2014 and 2015. Interviews were conducted onsite and via telephone. Where feasible, the researchers conducted follow-up interviews after the conclusion of the preceptorships. In Phase One, 13 participants (three nursing students, four medical students, two RNs, two MDs, one unit manager and one faculty member) took part in 18 one-on-one interviews and two focus groups. In Phase Two, 12 participants (two nursing students, two medical students, four RNs, one $\mathrm{MD}$, two unit managers and one faculty member) took part in 17 one-on-one interviews and two focus groups.

The researchers began coding the data as soon as the first round of interviews was transcribed. The researchers carried out 1) open coding, wherein they discovered innate patterns in the data and gathered it into nodes (or categories), until these nodes were saturated; and 2) theoretical coding, wherein they organized these nodes into themes, which they tested against the dataset to rationalize the patterns therein. This thematic analysis continued through to the validation stage.

\subsection{Validation}

Rigour was established through independent coding by three researchers, using both hardcopy and NVivo 10-11. Once coding was complete, the researchers compared their findings for mutual auditability and confirmability. The findings were validated through follow-up, semistructured interviews with the participants (where available), and onsite PowerPoint presentations, whereby the participants corroborated and legitimized the researchers' themes (Fereday \& Muir-Cochrane, 2006, p. 7). In these follow-up interviews and presentations, participants verified the findings through internal replication - namely the confirmation of their own data - and external replication - namely the corroboration of data from other sites.

\section{Findings}

Thematic analysis of interview and focus group data revealed that the IPRPP 1) induced all participants (students, preceptors, site managers and faculty) to reflect on tacit power dynamics between nursing and medicine, which they acknowledged to be in play regardless of the rural setting; 2) sensitized medical students to the practice scope and knowledge base 
of registered nurses and nursing students, whom they came to value as genuine collaborators and mentors; and 3) empowered nursing students to embrace more central, vocal roles in the IP team, and take the initiative in collaborating with their medical peers. Side-by-side IP interactions and rural clinical culture were instrumental to these outcomes.

The researchers derived three themes pertaining to the research question: 1) Us and them, denoting how the pilot provided an occasion for participants to reflect on cultural barriers to IPE and IP collaboration; 2) I didn't know a nurse did that, denoting the transformation of medical students' attitudes nursing knowledge and scope of practice, and willingness to capitalize on IP interactions to increase their own knowledge base; and 3) Feeling empowered, denoting the transformation of nursing students' assertiveness, initiative, and engagement in the IP team as equals.

\subsection{Us and Them}

The IPRPP induced all participants — students, preceptors, managers and faculty - to reflect on longstanding, tacit boundaries between nursing and medicine, young and old, and female and male. Attempting to traverse these boundaries could be uncomfortable, even in the smallest, most ostensibly relaxed settings, particularly when it came to longstanding matters of instructional etiquette between precepting RNs and MDs.

Those who did buy into the pilot surmised that discipline, length of practice experience and gender might be in play for those who seemed averse or disengaged. "I feel like my preceptor is vaguely aware of this project," remarked Stephanie, a medical student; "he doesn't ever mention it, like 'you should go get the nursing student'." Josephine, a nursing student, shared a particularly discouraging experience:

[The med student and I] did an assessment, which went really well, and then the two of us were supposed to come report to his preceptor, and let him know what our findings were. However, the medical student let me know via text that the physician didn't need to hear from me-that he got what he needed to know from the medical student. ...I was a little disheartened when we came out of that; we were supposed to go to speak to a physician together, and tell them what we thought...[but he] wasn't actually all that interested in what my thoughts were.

Stephanie, the medical student, was quick to defend her preceptor, saying, "It's no fault of his; I know he's busy, and that's the last thing on his mind when he has so many other things going on." But other students were more open to speculating on the culturally embedded power relations underlying MDs' coolness to the pilot. "I think maybe there was some wariness of bringing in new things, and ruffled feathers, and kind of messing with what people have as established routines," mused Fiona, another medical student at the same hospital, "just because that's the routine and the tradition in medicine."

The MDs' years of practice experience and gender were additional factors, not lost on the students. Said Rebecca, a nursing student placed at the same hospital, 
There are definitely doctors who have worked here longer, who have that tough doctor [attitude]: the nurse will tidy up after him, he has that authority type, [he] walks in with the authority. And then there are the younger doctors that come in, [who] are really like, Can I help you out with that, do you want me to put a sticker on this or did you guys just do it, and they're very open. So it is a bit of a generational thing too... I have worked a lot with Dr. Johnson and she's been really good with this. And I think that she's the only one that I have actually interacted with, regarding this study. She has emailed me personally, trying to organize some collaborations, and I've found her to be a good facilitator for this.

Rebecca did not overtly single out age and gender as determinants of MDs' behaviour. However, a gendered power discourse is readily apparent in the image of an older, "tough doctor" after whom "the nurse will tidy up," particularly when set against the example of a younger, female physician whose behaviour was quite the reverse. Rebecca went on to remark that "[Dr Johnson is] quite young, she's new, and I think this [pilot] is something she may value more [than] some of the other doctors."

Other students noted that buy-in seemed to depend on the individual MD. "The quality of the interprofessional component has a lot to do with the effort and personalities by some of the physicians," remarked Josephine, a nursing student. "If you're working with a physician who enjoys teaching and you know wants to include you in care, then you'll have a totally different day [than when] you're with a physician that doesn't get to know your name." Julie, another nursing student, agreed that the IPE experience was "very different" with different MDs. In taking stock of the nursing students' experiences, their faculty coordinator felt the "pinnacle" occupied by doctors "broke down more" at rural sites, but he acknowledged these sites were not immune to the traditional power discourse. "Maybe some doctors are more set in their ways, and maybe it's just the personality," he remarked. "Some people just have a personality where they're not quite as open, or they're a little bit more reserved, back into their own traditional structures."

The preceptors who did take part - both MDs and RNs - also reflected on cultural barriers to buying in. "Before this program, it was not my job or role to interfere with the nursing instruction process," remarked Melanie, an MD. "Whether [we're] territorial by design, or by default, I've never seen a nursing instructor instruct the medical students, and in return, I don't step over the line and take [nursing students] aside... there's a line and it's been well-defined." Melanie's description of territoriality 'by design or by default' speaks to a tacit boundary between nursing and medicine. She was furthermore ambivalent about this boundary, concluding "That's probably what's perpetuated some of the positive and negative things between the two professions." Marla, an $\mathrm{RN}$, made no attempt to conceal her disappointment with the pilot's first phase to date. "There is still this us and them... I thought we were supposed to try and get the team-building going, and I'm not really seeing a whole lot of it." 


\section{2 I Didn't Know a Nurse Did That}

Whatever misgivings participants expressed over some MDs' investment in the pilot, the medical students capitalized on opportunities to learn from the registered nurses and nursing students. "Some of the med students said, 'I didn't know a nurse did that'," recounted the nursing faculty coordinator. "They got that real, holistic sense, and I think that was really beneficial for them."

For the medical students, this holistic sense included a greater appreciation-which they vowed to carry forward into their professional practices-for nurses' scope of practice and knowledge base. Said Fiona, a medical student,

It's been really nice to just sit back and observe what the nursing students do-how they do their assessments. Oftentimes they might even be a bit more thorough than what we might initially do, so it's good to know their scope, and appreciate where they're coming from.... It seems foolish to turn your back to that, once you learn how much they have to offer, what they can bring to the table in terms of the holistic approach to patient care. ...A big part of [this experience] is breaking down some stereotypes and traditions, or ways of the past, and showing people that you're open to hearing what they have to say, and acknowledging them as other health professionals... also acknowledging our own limitations... hopefully [that] levels the playing field a little bit.

The pilot was an object lesson in humility. Acknowledging and overcoming "stereotypes and traditions"- even those perpetuated by some of their rural preceptors-was a major breakthrough for the medical students, borne of side-by-side experience with nurses and nursing students. David, a medical student, was impressed by nurses' "much more powerful role in terms of continuity of care - a lot of times nurses are with a patient for eight hours, half a day, or sometimes even longer; or you go to the ICU and it's like one-to-one." David also sought out RNs and nursing students to improve his knowledge of procedures: "In Emerg, I wanted to start a couple IVs and so some of the nurses were showing me how.... Also, one of the nursing students showed me how to inject into the buttocks, like an IM injection." Rashid, another medical student, admitted "there are a lot of things I don't know about medical care from the nursing perspective, and I think that's a really good learning experience for me. ...Nursing students could be more into [the patients'] immediate status, and what immediate problems they have. That really helps to orient a medical professional."

While some precepting MDs seemed too occupied or reluctant to play an active role in the pilot, others encouraged their students' initiative. "If the nursing student's there, I push the medical student [to] go talk to her or him," said Melanie (MD). "And if the nursing instructor or mentor is there, I try to let the medical student interact with them first, and then try to use that as a tool." Melanie stated elsewhere that she herself was unwilling to "step over the line" and instruct a nursing student, but she nonetheless saw her nursing colleagues and their students as a valuable educational resource. Her fellow physician Lori expressed a similar view: "the nursing students have lots to offer the medical students, in terms of procedures and what actually happens to patients behind the scenes, which is something the medical students 
sometimes don't realize." Other preceptors and staff members were also pleased to see medical students availing themselves of an underutilized trove of knowledge. Elaine, a site manager, felt this opportunity was a primary benefit of the pilot: "If some of the medical students are able to see just how much the nurse is able to do, and how it helps their roles when examining patients... it gives them a better perspective." Lucy, a precepting RN at the same site, corroborated this view from the nursing perspective: "Even though you're working with the med student, they're learning lots from you all the time... med students don't really understand what the nurses know, or what their limits are."

The medical students learned not only to value the knowledge of nurses and nursing students, but also to respect their roles as collaborators and team members. Justin, a medical student, recalled how he learned "to pick the brains of nurses and doctors, and other colleagues ... in a way that fuels my own learning." He hastened to point out, however, that his greatest gains were behavioural.

[It's] not just [that] we can actually learn clinical skills from [nurses], or some history stuff, or clinical pearls. ...if I know the different skills that different nurses or different kinds of nurses are bringing to the table, it [helps me to] communicate with them, to find out more information, to rely on their skills, to ask for their help, or to maybe ask them to take a lead on something.

Practising alongside nurses and nursing students taught Justin and the other medical students to acknowledge their own limits, to depend on others and to delegate responsibility. As Justin summarized it, "[this experience] primes me for the future, just so I can be a better team player."

\subsection{Feeling Empowered}

While the medical students spoke of their inroads in learning about procedures, nursing scope of practice, and teamwork, the nursing students characterized their gains primarily as psychosocial. The necessity of showing leadership and initiative, coupled with the esteem shown them by medical students and staff, translated into greater self-respect and confidence as they prepared to graduate. One factor instrumental to this outcome was the deliberate offsetting of the nursing and medical students' years of education. In pairing $4^{\text {th }}$ Year nursing students on their final practica with $3^{\text {rd }}$ Year medical students on a clinical rotation, the researchers set the nursing students up as the senior partners. Josephine, for example, remarked "[the med student is] still learning; he's $3^{\text {rd }}$ Year and there's certain things that he can do, but he can't write orders yet." For her part, she felt she was "more at the point, being $4^{\text {th }}$ Year, where I'm giving medications, carrying out physicians' orders, doing my assessments, and following through on treatments." She was surprised to discover that the medical student, a few years older than herself, did not yet know how to perform an ECG or an IV, whereupon she gladly acted as a clinical teacher. "We spent the day doing IVs together," she recalled. The faculty coordinator related an exchange with another nursing student participant who had guided a medical student through his first delivery. "The med student said something like, 'you seem like a pro at this,' and I think that made Courtney feel 
confident in her knowledge. She felt, wow, I can teach someone else." The pilot helped socialize the nursing students not only in the role of IP team members, but also as IP mentors.

Another empowering factor was a month-long head start given to nursing students, owing to differing preceptorship schedules between the Faculties of Nursing and Medicine. The extra time allowed the nursing students to acclimate as team members, and it reinforced their senior roles as guides and mentors to the incoming medical students. "It made for kind of a nice opportunity for me to settle in and sort of find my place. And right from the beginning, I felt like I was part of the team. It was very accepting," said Courtney, a nursing student. One site manager, Elaine, liked the fact that the nursing and medical students "weren't both new together; [the nursing student] was more familiar with our routines and how we worked, and I think she kind of directed the medical student at that point."

It often fell to the nursing students to take the lead on IPE experiences with their medical counterparts. "I would say that I have taken on the majority of the responsibility in terms of setting up the interaction," remarked Josephine, a nursing student. "It doesn't take a whole lot of effort to talk to him or whatnot, but I definitely have to; I do think that I take the initiative... to go forward and get the learning opportunity." With minimal support from a university faculty 100-400 km distant, the task of coordinating schedules and finding mutual, teachable moments could be challenging. "I think it has taken a bit more organization than I thought, in terms of time I've spent," said Rebecca, another nursing student. "I kind of pictured it being all laid out already, but we didn't know what it was gonna be like-no one knew." Indeed, the Phase One rollout of the pilot at the first site, which preceded the other two sites by 12 months, was beset with scheduling problems.

Succesful interactions often depended on the students' ingenuity. At one site, an impromptu huddle evolved from weekly Telehealth conferences (a video link providing distance learning and consultation for rural health care providers). "A lot of the nurses don't actually go to Telehealth; usually we're the only ones," Rebecca commented. "In real life the nurses can't always take that half hour, 45 minutes away from the unit, or they don't wanna use their breaks [for it]." For the students, however, Telehealth proved to be "a good opportunity for us to discuss the presentation and our perspectives from nursing, and from [the] medical students," as Julie, a nursing student, put it. She went on to add, "we took the initiative as students to organize the [primary clinic network] meetings as well; coming from [the city] I had no connections [here], so we had to create those, which was a good opportunity-[just] not what I was expecting." The medical students did not omit to give credit where it was due. "At the end of the day, my primary role is to successfully complete this rotation, and I have certain things I have to do, and I can't sacrifice those in lieu of doing the interprofessional things," admitted Stephanie. "[It's about] trying to carve out those opportunities, and... if you don't seek them out, they're not gonna happen. Rebecca [nursing student] could call me and say we had this opportunity, but I have to agree to come."

Just to be heard out by medical students and physicians was transformative for the nursing students. 'I've definitely had experiences on some acute urban settings where [there has] been fear, amongst my peers and I, of speaking up because of having witnessed horizontal 
violence in the workplace," said Courtney, a nursing student- "not necessarily bullying, but complete distrust and lack of communication amongst the different professions." Rebecca, a nursing student at a different site, concurred: "Sometimes nurses feel pressure talking to doctors, and nursing students get especially worked up sometimes...-Oh my god, I called a doctor-it's a big deal." The pilot prepared her to express herself to physicians because, in her words, she and the medical students "start on even ground; we're both learning and we're both vulnerable, and we can talk about things and not feel that we're talking up to someone." In many cases, egalitarian relationships with the medical students emboldened the nursing students to interact more directly with the medical preceptors. "Sometimes you do want to talk to the doctor, and then when they have a student or a resident, you're not sure what their role is; it's almost more intimidating," Rebecca continued, "but knowing that you're supposed to be... collaborating with the med students, it almost makes it easier to talk to the physician than it normally is."

The precepting physicians did not significantly adjust their routines for the sake of the pilot, whatever they felt about its objectives. Of greater consequence was their attitude toward the nursing students, which — with the few exceptions noted above-was more welcoming and encouraging than the students had expected. "It wasn't [that] he just wanted to speak to the RN and didn't care about the student's perspective," Courtney recalled. "It was very much we're a team, we need to learn together and most importantly, we need to provide the best care to the patient. I found that really, really rewarding." The nursing students' prior, urban rotations had made them wary of overstepping their bounds, but the rural physicians mostly accepted and even solicited their input. "I've only been there for a month, but it's definitely made a huge impact on feeling empowered, when you feel essentially respected by the physician because he's one of many people there making decisions," said Josephine. "My opinion is one of many opinions that are all going back and forth between the physicians. ...it makes everyone feel a little more empowered. It really does get to holistic care, essentially." In the view of one site manager, the pilot was easy enough to implement, "because we are a small site... even before this [pilot], we've always included [the medical students] in everything, and same with nursing." The intimacy of the site made inclusion both desirable and inevitable:

There isn't really a severe defined line - this is what the nursing student does, and this is what the medical student does-because we are small, [and] we try to make it all-inclusive. ...if something's happening that we think would be a good learning experience for [the students], we've always had the practice of including them.

(Sheila, Site Manager)

\section{Discussion}

The findings indicate that the IPRPP largely achieved its objectives of delivering IP practice experiences to nursing and medical students undertaking clinical preceptorships, and socializing these students into an IP practice culture. Moreover, all participants - students, staff and faculty-acknowledged the experience obliged them to confront the tacit, hierarchical discourse between nursing and medicine, and to reflect on their own attitudes and 
behaviours. The pilot thus promoted critical thinking and dialogue about power-a longstanding deficit in IPE curricula (Kuper \& Whitehead, 2012; Paradis \& Whitehead 2015). The participants occasionally experienced power differential between doctors and nurses as a barrier to IP interaction (Baker et al., 2011; Pecukonis, Doyle, \& Bliss, 2008; Whitehead, 2007). Student and staff accounts of the older MDs' disengagement from pilot are consistent with Becker, Hanyok and Walton-Moss's (2014) finding that older instructors with uniprofessional training are more turf-conscious and resistant to IP education, but this does not suggest these MDs were otherwise opposed to collaborative practice. The medical students, for their part, were eager to contribute to the pilot and to the research study, despite previous findings suggesting this cohort is inherently resistant to the goals of IPE and IP research (Horsburgh et al., 2006; Olson, 2015).

With regard to buy-in, gender was a revealing aspect of the pilot demographic. All the nursing students and preceptors $(n=11)$ were female, as were the three site managers (also practising RNs), while four of the six medical preceptors were male, as were three of the six medical students. This distribution speaks to the reality that both disciplines, especially nursing, have been slow to redress the traditional assignment of women to nursing and men to medicine (Seenandan-Sookdeo, 2012). That both female physicians bought in to the IPRPP, when three out of four male physicians did not, lends some support to the Rebecca's (nursing student) speculation that the pilot's objectives were more compelling for younger, female MDs than their older, male colleagues. Given that the traditional, medical hegemony tends to marginalize not only nurses, but women (Bell, Michalec, \& Arenson, 2014; Hart, 2015; Price, Doucet, \& Hall, 2014; Price, Hall, Angus, \& Peter, 2013), the female MDs perhaps embraced the pilot as a means of advancing both gender- and disciplinary parity. Thus, while lack of buy-in by more experienced, male MDs may have deprived some students of richer IP experiences, it also provoked fruitful and timely reflections about power and clinical culture, which added value to the pilot.

The IPRPP both capitalized on and established safe conditions for the construction of IP identity, thereby serving a key environmental component in Khalili et al.'s (2013) programme of interprofessional socialization (IPS). The "pragmatic hierarchy" (Burford et al., 2013, p. 397), wherein experienced nurses mentor novice physicians, accurately characterizes the relationship between the nursing and medical students. In acknowledging their nursing counterparts' complementary (and at turns deeper) knowledge base and scope of practice, the medical students willingly abjured any stereotypical notions of medical superiority (Horsburgh et al., 2006; Page \& Meerabeau, 2004). Where Burford et al. (2013) view the pragmatic hierarchy as a temporary phenomenon, however, participants in this study regarded it as a foundation for future practice, in which a level playing field would be the rule and not the exception.

Establishing a level playing field between nursing and medical students was partly a matter of timing, insofar as nursing students had the advantages of seniority and advance placement onsite, but it was also a matter of space. Much as nurses and doctors rely on informal, liminal spaces, such as corridors, to exchange information unhindered by disciplinary boundaries (Bleakley, 2013), students at one site discovered an ideal space for informal exchanges and 
planning in the Telehealth Room. Generally speaking, the rural health care sites in the study were much less "striated" (Bleakley, 2013, p. 27) than urban sites, having fewer discrete units and more shared space. The findings suggest that a culture of IP boundary-crossing, social empowerment and holistic care grows in part from the communal, liminal nature of these smaller rural settings (Hays, 2008).

The conjunction of rural, clinical context, IP experience and a pre-professional stage of education was unique to this pilot, but it built on an established practice of experiential, side-by-side IP training (Hallin, Kiessling, Waldner, \& Henriksson, 2009; Pecukonis, Doyle, \& Bliss, 2008; Weller, Boyd, \& Cumin, 2014). Crucially, the participants understood that their gains - increased sensitivity to stereotypes, respect for colleagues' voices, and personal empowerment — were not ends in themselves, but elements of a team-based safety culture and a holistic centering of the patient (Margalit et al., 2009; Price, Doucet, \& Hall, 2014; Weller, Boyd, \& Cumin, 2014; WHO, 2010).

\subsection{Implications for Further Practice}

Rural acute care sites have much to offer experiential IPE: a collaborative, patient-centered culture borne of an intimate physical setting, a generalist perspective, and holistic continuity of care. Researchers are well advised to temper their expectations of physician buy-in, however, particularly when IPE initiatives involve a qualitative research component. Coolness to IPE does not necessarily mean opposition to collaborative practice, or the desire to protect one's turf, although some rural physicians do live up this traditional stereotype. The partnering of senior nursing students with mid-program medical students fosters a sense of educational parity between the two disciplines, encouraging medical students to seek out nursing knowledge and nursing students to be more assertive, vocal IP team members.

\subsection{Limitations}

Students choosing rural placements are a small cohort in any health science discipline, necessarily limiting the sample of students and preceptors available for recruitment, either through purposive or snowball sampling. Had more MDs who volunteered for the pilot taken part in interviews, focus groups or validation sessions, the researchers might have achieved a yet more representative and rigorous dataset. The study does not reveal anything about the potential for an urban-based pilot, beyond participants' impressions that they witnessed less collaborative practice in their urban rotations. The findings pertain not only to nursing and medicine, but how future IPE initiatives could involve other disciplines such as pharmacy, occupational therapy, and social work.

\section{Conclusion}

Silos, tribes and turf protection persist in health care settings large and small, urban and rural. To have maximum effect, IPE must offer collaborative, hands-on, team experiences to students and novice practitioners, before they become socialized into a lopsided, physicianand male-dominated hierarchical discourse that has held sway for generations of health care 
providers. Experiential, practice-based programs enable participants to confront, reflect upon, and adjust their attitudes and behaviours in response to this hierarchical discourse. Such programs only succeed when participants buy in and capitalize on opportunities to learn, teach, and collaborate; but designers of IPE initiatives can do their part to level the playing field for participants, by optimizing aspects of timing and space. A rural care setting with exemplary team ethos and patient safety culture is a good starting point.

\section{Acknowledgement}

Funding for the study was provided by [removed for blind peer review]. The authors wish to acknowledge [removed for blind peer review], Research Assistant, for his role in collecting data and in the writing of this article.

\section{References}

Baker, L., Egan-Lee, E., Martimianakis, M., \& Reeves, S. (2011). Relationships of power: Implications for interprofessional education. Journal Of Interprofessional Care, 25(2), 98-104. http://dx.doi.org/10.3109/13561820.2010.505350

Becker, K. L., Hanyok, L. A., \& Walton-Moss, B. (2014). The turf and baggage of Nursing and Medicine: Moving forward to achieve success in interprofessional education. Journal For Nurse Practitioners, 10(4), 240-244. http://dx.doi.org/10.1016/j.nurpra.2014.02.004

Bell, A. V., Michalec, B., \& Arenson, C. (2014). The (stalled) progress of interprofessional collaboration: The role of gender. Journal Of Interprofessional Care, 28(2), 98-102. http://dx.doi.org/10.3109/13561820.2013.851073 (n.d).

Bleakley, A. (2013). The dislocation of medical dominance: Making space for interprofessional care. Journal of Interprofessional Care, 27(S2), 24-30. http://dx.doi.org/10.3109/13561820.2013.791672

Burford, B., Morrow, G., Morrison, J., Baldauf, B., Spencer, J., Johnson, N., \& ... Illing, J. (2013). Newly qualified doctors' perceptions of informal learning from nurses: Implications for interprofessional education and practice. Journal Of Interprofessional Care, 27(5), 394-400. http://dx.doi.org/10.3109/13561820.2013.783558

Fereday, J. \& Muir-Cochrane, E. (2006). Demonstrating rigor using thematic analysis: A hybrid approach of inductive and deductive coding and theme development. International Journal of Qualitative Methods, 5(1), 1-13. https://doi.org/10.1177/160940690600500107

Hallin, K., Kiessling, A., Waldner, A., \& Henriksson, P. (2009). Active interprofessional education in a patient based setting increases perceived collaborative and professional competence.

Medical

Teacher,

$31(2)$,

151-157. 
https://doi.org/10.1177/160940690600500107

Hart, C. (2015). The elephant in the room: Nursing and nursing power on an interprofessional team. Journal of Continuing Education In Nursing, 46(8), 349-355. http://dx.doi.org/10.3928/00220124-20150721-01

Hays, R. (2008). Interprofessional education in rural practice: How, when and where? Rural and Remote Health, 8(939), 5.

Horsburgh, M., Perkins, R., Coyle, B., \& Degeling, P. (2006). The professional subcultures of students entering medicine, nursing and pharmacy programmes. Journal of Interprofessional Care, 20(4), 425-431. https://doi.org/10.1177/160940690600500107

Khalili, H., Orchard, C., Spence Laschinger, H. K., \& Farah, R. (2013). An interprofessional socialization framework for developing an interprofessional identity among health professions students. Journal of Interprofessional Care, 27(6), 448-453. http://dx.doi.org/10.3109/13561820.2013.804042

Kuper, A., \& Whitehead, C. (2012). The paradox of interprofessional education: IPE as a mechanism of maintaining physician power?. Journal Of Interprofessional Care, 26(5), 347-349. http://dx.doi.org/10.3109/13561820.2012.689382

Margalit, R., Thompson, S., Visovsky, C., Geske, J., Collier, D., Birk, T., \& Paulman, P. (2009). From professional silos to interprofessional education: Campuswide focus on quality of care. Quality Management In Health Care, 18(3), 165-173. http://dx.doi.org/10.1097/QMH.0b013e3181aea20d

McNair, R. (2005). The case for educating health care students in professionalism as the core content of interprofessional education. Medical Education, 39(5), 456-464. https://doi.org/10.1111/j.1365-2929.2005.02116.x

Olson, R. (2015). How would an egalitarian health care system operate? Power and conflict in interprofessional education. Medical Education, 49(4), 353-354. http://dx.doi.org/10.1111/

medu. 12686

Page, S., \& Meerabeau, L. (2004). Hierarchies of evidence and hierarchies of education: Reflections on a multiprofessional education initiative. Learning In Health \& Social Care, 3(3), 118-128. https://doi.org/10.1111/j.1473-6861.2004.00070.x

Paradis, E., \& Whitehead, C. R. (2015). Louder than words: Power and conflict in interprofessional education articles, 1954-2013. Medical Education, 49(4), 399-407. http://dx.doi.org/10.1111/medu.12668

Pecukonis, E., Doyle, O., \& Bliss, D. (2008). Reducing barriers to interprofessional training: Promoting interprofessional cultural competence. Journal of Interprofessional Care, 22(4), 417-428. https://doi.org/10.1080/13561820802190442

Price, S., Doucet, S., \& Hall, L. M. (2014). The historical social positioning of nursing and 
medicine: Implications for career choice, early socialization and interprofessional collaboration. Journal of Interprofessional Care, 28(2), 103-109. http://dx.doi.org/10.3109/13561820.2013. 867839

Price, S., Hall, L. M., Angus, J., \& Peter, E. (2013). The social context of career choice among millennial nurses: Implications for interprofessional practice. Journal of Interprofessional Care, 27(6), 509-514. http://dx.doi.org/10.3109/13561820.2013.816660

Reeves, S., Perrier, L., Goldman, J., Freeth, D., \& Zwarenstein, M. (2013). Interprofessional education: effects on professional practice and healthcare outcomes (update). Cochrane $\begin{array}{llll}\text { Database } & \text { of } & \text { Systematic } & \end{array}$ http://dx.doi.org/10.1002/14651858.CD002213.pub3

Seenandan-Sookdeo, K. I. (2012). The influence of power in the Canadian healthcare system. Clinical Nurse Specialist: The Journal for Advanced Nursing Practice, 26(2), 107-112.

Weller, J., Boyd, M., \& Cumin, D. (2014). Teams, tribes and patient safety: Overcoming barriers to effective teamwork in healthcare. Postgraduate Medical Journal, 89(1061), 149-154. http://dx.doi.org/10.1136/postgradmedj-2012-131168

Whitehead, C. (2007). The doctor dilemma in interprofessional education and care: How and why will physicians collaborate?. Medical Education, 41(10), 1010-1016. https://doi.org/10.1111/j.1365-2923.2007.02893.x

World Health Organization (WHO) (2010). Framework for action on interprofessional education and collaborative practice. Geneva: World Health Organization.

\section{Copyright Disclaimer}

Copyright for this article is retained by the authors, with first publication rights granted to the Journal of Sociological Research.

This is an open-access article distributed under the terms and conditions of the Creative Commons Attribution license (http://creativecommons.org/licenses/by/3.0/). 